\title{
lonospheric response over Europe during the solar eclipse of March 20, 2015
}

\author{
Mohammed Mainul Hoque*, Daniela Wenzel, Norbert Jakowski, Tatjana Gerzen, Jens Berdermann, \\ Volker Wilken, Martin Kriegel, Hiroatsu Sato, Claudia Borries, and David Minkwitz \\ German Aerospace Center (DLR), Institute of Communications and Navigation, Kalkhorstweg 53, 17235 Neustrelitz, Germany \\ *Corresponding author: mainul.hoque@dlr.de
}

Received 23 March 2016 / Accepted 5 September 2016

\begin{abstract}
The solar eclipse on March 20, 2015 was a fascinating event for people in Northern Europe. From a scientific point of view, the solar eclipse can be considered as an in situ experiment on the Earth's upper atmosphere with a well-defined switching off and on of solar irradiation. Due to the strong changes in solar radiation during the eclipse, dynamic processes were initiated in the atmosphere and ionosphere causing a measurable impact, for example, on temperature and ionization. We analyzed the behavior of total ionospheric ionization over Europe by reconstructing total electron content (TEC) maps and differential TEC maps. Investigating the large depletion zone around the shadow spot, we found a TEC reduction of up to 6 TEC units, i.e., the total plasma depletion reached up to about 50\%. However, the March 20, 2015 eclipse occurred during the recovery phase of a strong geomagnetic storm and the ionosphere was still perturbed and depleted. Therefore, the unusual high depletion is due to the negative bias of up to $20 \%$ already observed over Northern Europe before the eclipse occurred. After removing the negative storm effect, the eclipse-induced depletion amounts to about $30 \%$, which is in agreement with previous observations. During the solar eclipse, ionospheric plasma redistribution processes significantly affected the shape of the electron density profile, which is seen in the equivalent slab thickness derived by combining vertical incidence sounding (VS) and TEC measurements. We found enhanced slab thickness values revealing, on the one hand, an increased width of the ionosphere around the maximum phase and, on the other, evidence for delayed depletion of the topside ionosphere. Additionally, we investigated very low frequency (VLF) signal strength measurements and found immediate amplitude changes due to ionization loss at the lower ionosphere during the eclipse time. We found that the magnitude of TEC depletion is linearly dependent on the Sun's obscuration function. By modelling TEC depletion and knowing the Sun's obscuration function in advance, Global Navigation Satellite System (GNSS) operators may improve the broadcast ionospheric correction during a solar eclipse day.
\end{abstract}

Key words. Ionosphere (general) - Total electron content - Remote sensing - Space weather - Ionization

\section{Introduction}

A solar eclipse is a natural phenomenon that occurs when the moon appears between the Sun and the Earth, and the moon fully or partially blocks the Sun, casting a shadow over the Earth. Although a total solar eclipse is a rare event, it can occur somewhere on Earth every 18 months on average. Since the moon's shadow moves rapidly from west to east across the Earth at supersonic speed, the total eclipse lasts for only a few minutes (up to $7 \mathrm{~min}$ ) at any location (https://en.wikipedia.org/ wiki/Solar_eclipse and references therein). However, its effect on the ionosphere persists even for hours after the maximum obscuration. The solar eclipse on March 20, 2015 was a fascinating event for people in Northern Europe. The eclipse occurred from 8:00 to 11:40 Universal Time (UT), moving from North-West toward North-East Europe. Outside the totality zone, the shadow of the eclipse was still visible to varying degrees across the continent. Generally speaking, the attenuation of solar irradiation and thermospheric heating during a solar eclipse leads to thermospheric/ionospheric cooling and plasma depletion at ionospheric heights.

Since most solar eclipses occur in mid- and low latitudes (e.g., Jakowski et al. 1983, 1999, 2001, 2008; Cheng et al. 1992; Fritts \& Luo 1993; Mueller-Wodarg et al. 1998;
Krankowski et al. 2008; Le et al. 2008, 2009, 2010), the number of publications considering high-latitude eclipses is rather low (e.g., Rashid et al. 2006; Pitout et al. 2013). Le et al. (2009) investigated the latitudinal dependence of the ionospheric response to solar eclipses and found that eclipse effects are larger at middle latitudes than at low latitudes. However, above $40^{\circ} \mathrm{N}$ latitude, the eclipse effect decreases with increasing latitude. Whereas the analysis of low-latitude eclipses is affected by dynamic processes related to the equatorial anomaly (e.g., Cheng et al. 1992; Le et al. 2010), high-latitude eclipses are affected by a strong coupling with the magnetosphere, in particular during geomagnetic storms (e.g., Rashid et al. 2006; Pitout et al. 2013). The analysis of the solar eclipse on March 20, 2015 presented here will contribute to comparing solar eclipse effects at different latitudes. As shown in many publications, the depletion of total electron content (TEC) during a total eclipse at mid-latitudes is about $30-40 \%$ with a delay from 20 to 40 min (e.g., Jakowski et al. 2001, 2008; Krankowski et al. 2008). Simultaneous ionosonde measurements of peak electron density NmF2 enable estimates of the equivalent slab thickness $\tau$ by computing the ratio $\tau=\mathrm{TEC} / \mathrm{NmF} 2$. This quantity, which describes the width of the vertical electron density profile, is mostly enhanced during eclipses, although thermospheric cooling 
should cause a contraction of the thermosphere-ionosphere system, i.e., a decrease of slab thickness (e.g., Cohen 1984; Jakowski et al. 2001, 2008). The observations indicate that a number of competitive processes are initiated by an eclipse often enhanced by dynamic forces associated with large-scale geophysical conditions not directly impacted by the solar eclipse. Thus, the ionospheric depletion signatures may be heavily disturbed by electrodynamic forces in equatorial regions (e.g., Cheng et al. 1992; Tsai \& Liu 1999) and by space weather effects, such as particle precipitation, at high latitudes (e.g., Rashid et al. 2006; Pitout et al. 2013). As reported by Chimonas \& Hines (1970) and others, the rapidly moving cooling spot can act as a continuous source of travelling ionospheric disturbance (TID) like gravity waves (e.g., Liu et al. 1998; Altadill et al. 2001; Jakowski et al. 2008). On the other hand, the origin of TIDs observed during eclipses often remains unclear due to enhanced geomagnetic activity or other perturbations like strong weather fronts in the troposphere (Jakowski et al. 2001, 2008). Hence, considering the fact that a few days before the eclipse occurred on March 20, there was a severe geomagnetic storm, it is difficult to distinguish between TIDs that might have been caused by the solar eclipse or by storm-induced perturbations in the recovery phase (Verhulst et al. 2016). However, Verhulst et al. (2016) found signatures of TIDs during the solar eclipse in question in high-resolution ionospheric observations over Belgium when analyzing ionospheric tilt and plasma drift measurements.

To understand the competing processes in more detail, physics-based modelling and simulation studies are required (e.g., Mueller-Wodarg et al. 1998; Pitout et al. 2013). It thus becomes clear that besides thermospheric cooling and composition changes, neutral winds should also be considered when explaining the observations. Before starting physics-based modelling of an eclipse, in this paper we initially discuss multi-sensor observations made during the solar eclipse. We present vertical sounding or ionosonde data characterizing key ionospheric parameters, such as peak electron density of the F2 layer NmF2 and the associated height hmF2. In combination with TEC measurements, the related slab thickness $\tau$ as mentioned above is estimated and discussed. As former studies have shown, total electron content is closely related to solar illumination (e.g., Cohen 1984; Jakowski et al. 2001, 2008). Since TEC represents the total ionization per square meter, compared with $\mathrm{NmF} 2$, this quantity is less sensitive to vertical plasma redistribution that might be initiated by external perturbation forces of non-eclipse origin.

Due to the close coupling of the photoionization processes with solar illumination, the ionization level follows the obscuration function in a certain way. Since a solar eclipse reduces the ionization of the overall ionosphere, it changes propagation conditions for very low frequency (VLF) signals. Because of their broad wavelengths, the VLF signals are reflected from the bottom of the ionosphere at the $\mathrm{D}$ layer around $70-80 \mathrm{~km}$ altitude. Due to the eclipse-induced reduction of photoionization, the D layer slowly disappears and the bottomside of the ionosphere moves upward, causing significant changes in signal strength and phase of VLF measurements. It is expected that the bottomside ionosphere moves upward causing an enlargement of the VLF ray path or time delay that has been reported in the literature (e.g., Cheng et al. 1992; Clilverd et al. 2001; Pal et al. 2012).

The spatial and temporal variations of the induced ionospheric changes are closely correlated with the obscuration function of solar radiation. Since the eclipse-initiated processes are time-consuming, we expect a delayed response to the obscuration function, increasing with altitude of the ionospheric layer (e.g., Jakowski et al. 2001, 2008). Moreover, plasma depletion also depends on the solar incidence angle that has to be considered when interpreting the observational data.

Since Global Navigation Satellite Systems (GNSS) use trans-ionospheric signals, the technical performance of applications might be seriously affected during a solar eclipse. When travelling through the ionosphere, the navigation signals are delayed due to interaction with ionospheric plasma. The ionospheric propagation delay is directly proportional to TEC and considered to cause the largest error in singlefrequency operations of GNSS. To aid single-frequency operation, the Global Positioning System (GPS)/Galileo transmits ionospheric correction coefficients as part of the satellite message on a daily basis. The correction coefficients are produced using TEC data from the previous day; this means the given corrections are predictions $24 \mathrm{~h}$ ahead of time (Hoque \& Jakowski 2014). During a solar eclipse, sudden plasma depletion occurs in the ionosphere and the ionospheric prediction which is computed based on TEC data of the previous day may not provide expected correction for singlefrequency GNSS users. Our investigation shows that the magnitude of TEC depletion is dependent on the Sun's obscuration function. Therefore, knowing the Sun's obscuration function in advance, GNSS operators may improve the broadcast ionospheric correction during a solar eclipse day.

In the present paper, we analyze the March 20, 2015 solar eclipse effects on the ionospheric structure over Europe based on VS (vertical incidence sounding), GPS, and VLF measurements. Whereas the VS measurements are used to provide peak electron density information at the F2, F1, and E layers over selected ionosonde stations, a dense network of GPS stations was used to provide higher spatial resolution of integral TEC data over Europe. We investigated the original TEC maps and differential TEC maps obtained by subtracting 27 day medians from the actual TEC map values on March 20, 2015. By combining VS and GPS measurements, the equivalent slab thickness was estimated over the ionosonde stations to obtain information on how the shape of the vertical electron density profile changes during the eclipse. To collect information on the bottomside ionosphere, the strength of VLF signals received at the German Aerospace Center (DLR), Neustrelitz from 6 Navy stations was analyzed. Additionally, the plasma depletion dependency on the obscuration function was compared with observations from the solar eclipse of October 3 , 2005.

\section{Data sources and data processing}

\subsection{TEC data}

The GNSS satellites orbit at about 20,000 km height above the Earth's surface and the received signals on the ground propagate through the ionosphere. They can effectively be used to estimate ionospheric TEC, which is an integral measure of electron density. Here, we used dual-frequency GPS observations to derive TEC. To investigate the ionospheric response over Europe during the solar eclipse of interest, we selected a period of 27 days from March 7 to April 2, 2015 (day of year, doy 66-92). The days were selected in such a way that the eclipse day on March 20 (doy 79) became the median day. The GPS dual-frequency precise code (P1 \& P2 signals) and carrier-phase measurements (L1 \& L2 signals) with $1 / 30 \mathrm{~Hz}$ 
sampling rate from about 175 globally distributed International GNSS Service (IGS; Dow et al. 2009) stations were used for global TEC reconstruction. About 50 GPS stations were available in the considered European region distributed over $30-80^{\circ} \mathrm{N}$ latitude and $30^{\circ} \mathrm{W}-40^{\circ} \mathrm{E}$ longitude. The data were downloaded from NASA's Crustal Dynamics Data Information System (CDDIS) archive (Noll 2010; ftp://cddis.gsfc.nasa.gov/ gnss/data/hourly/). The daily GPS ephemeris data were downloaded from the Scripps Orbit and Permanent Array Center (SOPAC) archive (http://sopac.ucsd.edu/cgi-bin/ dbDataByDate.cgi). The differential phase and code measurements were used to derive ionospheric TEC. However, the differential phase and code measurements suffer from phase ambiguities, the receiver and satellite inter-frequency biases. The details about ambiguity removal, TEC calibration, and separation of inter-frequency satellite and receiver biases are given in Jakowski et al. (2011). Thus, the TEC at numerous IGS stations was estimated for receiver-satellite link geometries having elevation angles $\geq 10^{\circ}$. We used the thin-shell ionosphere mapping function for slant to vertical TEC conversion and computed grid vertical TEC maps by assimilating station TEC into the background Neustrelitz TEC Model (NTCM; Jakowski et al. 2011). The geographic latitude and longitude resolutions were fixed at 2.5 and $5^{\circ}$, respectively, with 5 min time resolution.

In the present paper, we analyze the eclipse effects on the ionospheric structure over Europe and investigate the dependency of TEC depletion on the obscuration function during the recent solar eclipse. To verify whether the observed relationship is valid for other solar eclipse events, we selected the previous solar eclipse of October 3, 2005. Therefore, we processed the GPS data for the October 3, 2005 event as well as data from 13 days before and after the eclipse day (i.e., doy 262-289) and produced gridded TEC maps with similar spatial resolution as done for the year 2015 .

\subsection{Vertical sounding data}

The VS measurements were used to analyze the solar eclipse effect on the plasma frequencies foF2, foF1, and foE, which correspond to the peak electron densities at the F2, F1, and $\mathrm{E}$ layers, respectively. In addition, the F2 layer peak electron density height $\mathrm{hmF2}$ was analyzed. The VS data from the stations at Ebro/Spain (EB040, 40.8 $\left.\mathrm{N}, 0.5^{\circ} \mathrm{E}\right)$ and Dourbes/Belgium $\left(\mathrm{DB} 049,50.1^{\circ} \mathrm{N}, 4.6^{\circ} \mathrm{E}\right)$ were used in the present study. Both observatories performed measurements at a 5 min sampling rate. However, at the Dourbes station, the sampling rate was increased from $5 \mathrm{~min}$ to $1 \mathrm{~min}$ during eclipse hours 6:30-11:45 UT. The data were measured in Global Ionospheric Radio Observatory (GIRO) sites operated by the University of Massachusetts Lowell (UML) and we collected the data from the Lowell Digital Ionogram DataBase (Reinisch \& Galkin 2011; DIDBase, http://giro.uml.edu/). GIRO sites are equipped with digisonde instruments for probing the bottomside ionosphere from $80 \mathrm{~km}$ up to the peak of ionospheric plasma density.

The solar eclipse over Ebro started at 8:13 UT and ended at 10:05 UT, with the maximum obscuration occurring at 9:12 UT. The solar eclipse over Dourbes started at 8:32 UT and ended at 10:32 UT, with the maximum obscuration occurring at 9:34 UT.

The vertical sounding data were then combined with corresponding TEC data to derive the equivalent slab thickness $\tau$ defined by $T E C / N m F 2$, where the peak electron density
$\mathrm{NmF} 2$ is derived by $N m F 2=1.24 \times 10^{-2}(f o F 2)^{2}$ in the International System (SI) of units. The slab thickness $\tau$ is measured in $\mathrm{m}$ (converted to $\mathrm{km}$ ) when TEC is measured in electrons $/ \mathrm{m}^{2}$ and $N m F 2$ in electrons $/ \mathrm{m}^{3}$. As mentioned before, the derived equivalent slab thickness $\tau$ is a powerful measure of the shape of the vertical electron density profile, which may change dramatically during ionospheric perturbations.

\subsection{VLF data}

The VLF (3-30 kHz) and LF (low frequency, 30-300 kHz) transmissions facilitate long range communications over land, and over and under oceans/seas, and have been used mainly in the North Atlantic and Arctic regions where auroral and polar disturbances hamper reliable communication. The United States Navy has been using VLF transmissions for general broadcasting to ships/submarines as a means of communication and/or timing signal for many decades. Very recently, the DLR has installed a network of VLF receivers called Global Ionospheric Flare Detection System (GIFDS) for detecting solar flare occurrence by continuously monitoring VLF signals of the dayside ionosphere (Wenzel et al. 2016). GIFDS uses a Perseus SDR (Software Defined Radio) receiver (see Wenzel et al. 2016 and references therein for details) to receive several frequency channels (Navy stations) between 20 and $500 \mathrm{kHz}$. For the present analysis, we used data recorded at the receiving site DLR Neustrelitz (NZ). The broadcast Navy stations, their location and frequency of the received signals are listed in Table 1.

As mentioned in the table, the NZ station received VLF signals from 6 Navy stations namely NRK, GXH, GYN, FTA63, FUG, and TBB. Different propagation paths show different stages of obscuration during the solar eclipse. Since the exact reflection point at the bottomside ionosphere is not known, as an approximation, we computed mid-reflection points on the great circle for each propagation path. The stations NRK, GXH, and GYN are closely located to the eclipse path and the associated mid-reflection points suffer maximum obscuration of about $97 \%, 87.6 \%$, and $84.1 \%$, respectively. The solar eclipse roughly started approximately at 8:40 UT and ended at 10:40 UT at those reflection points with the maximum obscuration occurring at 9:42, 9:43, and 9:40 UT, respectively. The solar eclipse over FTA63 (at reflection point) started approximately at 8:38 UT and ended at 10:38 UT with the maximum obscuration of about $77.3 \%$ occurring at 9:39 UT. The station TBB is located farthest compared to other stations from the eclipse path and the maximum obscuration found as $51.2 \%$ at the mid-reflection point. It is worth mentioning that the solar eclipse over the NZ station started approximately at 8:38 UT and ended at 10:38 UT, with the maximum obscuration of about $73.1 \%$ occurring at the 9:39 UT.

\subsection{Sun's obscuration data}

The Sun's obscuration data were computed via the PyEphem application, which provides a Python package for performing high-precision astronomy computations (http://rhodesmill.org/ pyephem/). The original Astronomical Software Ephemeris (XEphem) was written by Elwood Charles Downey (http:// www.clearskyinstitute.com/xephem/). The application provides the positions of the Sun and Moon as well as their angular separation for a given date and location on the Earth's surface. The obscuration values are calculated considering their overlapping, as seen from the Earth at a specific location and 
Table 1. Received Navy stations, their location and frequency of the VLF signals.

\begin{tabular}{lccccccc}
\hline \hline $\begin{array}{l}\text { Frequency } \\
(\mathrm{kHz})\end{array}$ & $\begin{array}{c}\text { Call } \\
\text { sign }\end{array}$ & Latitude & Longitude & $\begin{array}{c}\text { Distance } \\
(\mathrm{km})\end{array}$ & $\begin{array}{c}\text { Max. obscuration at mid- } \\
\text { reflection }\end{array}$ & $\begin{array}{c}\text { Max. amplitude } \\
(\mathrm{dB})\end{array}$ & $\begin{array}{c}\text { Time of max. signal } \\
\text { fluctuation }\end{array}$ \\
\hline 37.5 & NRK & 63.85 & -22.47 & 2322.95 & $09: 42$ UT 97.0\% & 6.66 & $09: 42$ UT \\
57.4 & GXH & 58.59 & -3.63 & 1186.77 & $09: 43$ UT $87.6 \%$ & 7.97 & $09: 43$ UT \\
81.0 & GYN & 53.83 & -2.83 & 1048.66 & $09: 40$ UT $84.1 \%$ & 6.55 & $09: 42$ UT \\
63.85 & FTA63 & 48.54 & 2.58 & 907.08 & $09: 39$ UT 77.3\% & 1.61 & $09: 45$ UT \\
62.6 & FUG & 43.39 & 2.10 & 1369.29 & $09: 34$ UT 73.1\% & 3.98 & $09: 45$ UT \\
26.7 & TBB & 37.41 & 27.32 & 2084.28 & $09: 53$ UT $51.2 \%$ & 1.16 & $09: 56$ UT \\
\hline
\end{tabular}

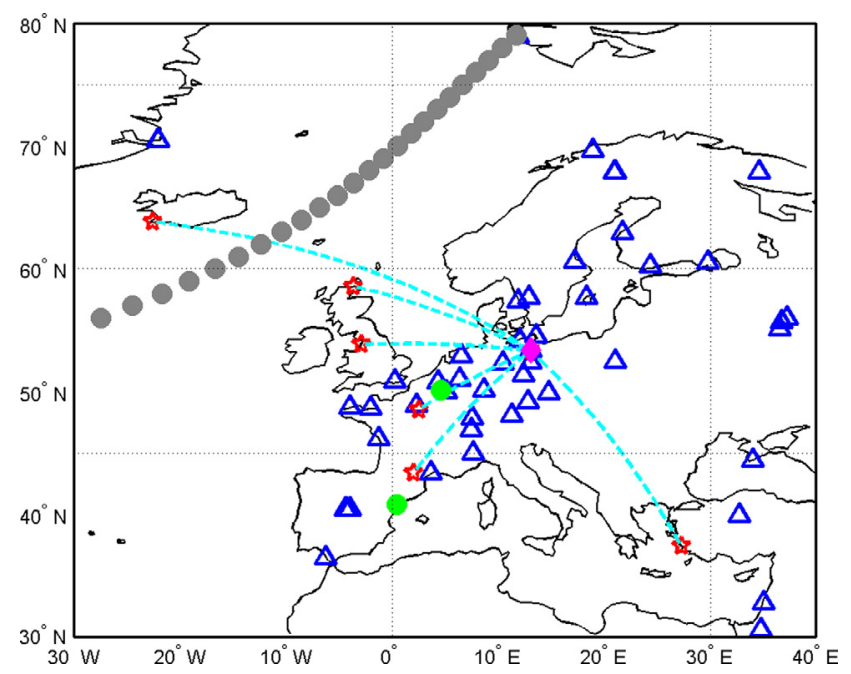

Fig. 1. Geographical distribution of the GPS receivers (blue triangle), ionosonde stations (green circle), Navy stations (red pentagon), and VLF receiver (magenta diamond). The gray colored circle line indicates the path of the eclipse shadow of the obscuration at ground level.

time. Maps of the obscuration were generated over a grid of $1^{\circ}$ times $1^{\circ}$ in latitude and longitude for the European area every $2 \mathrm{~min}$. The maximum obscuration values were taken from such maps. However, the obscuration values for different measuring sites were calculated separately with a time resolution of $1 \mathrm{~min}$.

The geographical distribution of the GPS receivers, ionosonde stations, and Navy stations, and VLF receiver used are shown in Figure 1. The daily solar radio flux F10.7 and the three-hourly geomagnetic activity index Kp during the selected periods (doys 66-92) are plotted in Figure 2. The F10.7 and Kp data are obtained from the Space Physics Interactive Data Resource (SPIDR) of NOAA's National Geophysical Data Center (NGDC). The F10.7 value was 111.8 flux units (sfu, $1 \mathrm{sfu}=10^{-22} \mathrm{Wm}^{-2} \mathrm{~Hz}^{-1}$ ) on the eclipse day, whereas the 27 day mean value found was $124.5 \mathrm{sfu}$. The maximum Kp index was found to be 4.7 on the eclipse day and 5.0, 6.0, and 7.7 during the previous three days, doy 78, 77, and 76, respectively. Therefore, although the eclipse day shows a moderate $\mathrm{Kp}$ value, the previous days show higher $\mathrm{Kp}$ values indicating significant geomagnetic activities.

\section{Data analysis and discussion}

\subsection{TEC measurements}

Since TEC is an integral measure of ionospheric electron density, reconstructed vertical TEC can provide accurate

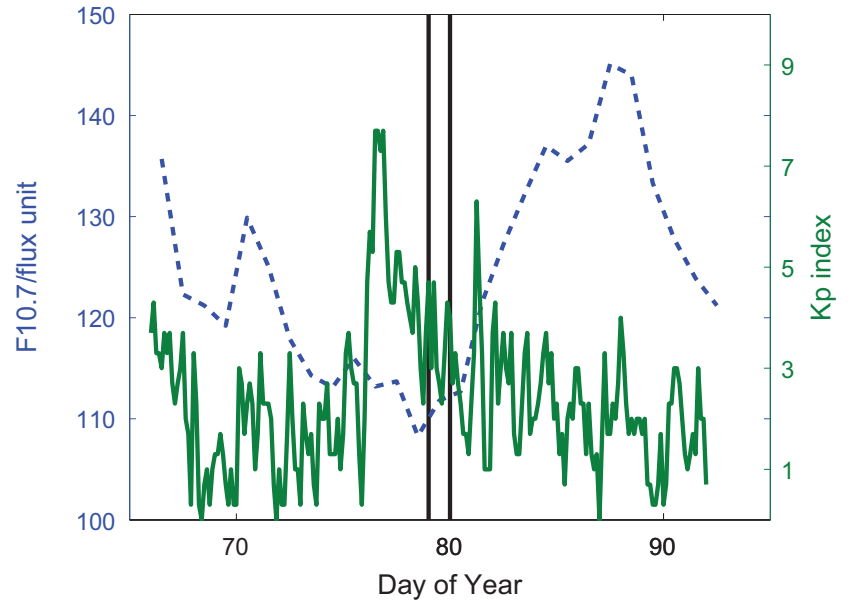

Fig. 2. Solar radio flux F10.7 and geomagnetic activity index Kp variations before and after the solar eclipse day on March 20, 2015 marked by a pair of black vertical lines.

information about total ionization loss as a result of reduced photoionization during a solar eclipse and thus corresponds better to the solar radiation function. TEC maps are generated combining data from distributed GPS stations and therefore local effects are smoothed out. However, sequential TEC maps enable us to study the horizontal distribution and transport of ionospheric plasma. Figure 3 a shows snapshots of reconstructed TEC maps over the European region $\left(40-80^{\circ} \mathrm{N}\right.$, $\left.40^{\circ} \mathrm{W}-40^{\circ} \mathrm{E}\right)$ for time instances of $8: 38,9: 38,10: 08$, and 10:38 UT on the solar eclipse day March 20, 2015. The filled black circle shows the exact geographic location where the maximum obscuration occurred at the mentioned time instance. Comparing with corresponding 27 day median maps (Fig. 3b), we see that the regular longitudinal TEC variation is perturbed by the sudden switching off of solar radiation during the eclipse passes. Consequently, we found wave-like signatures in the reconstructed TEC maps and found TEC depletion of up to a few (1-6) TEC units. Figure 3a shows the passes of maximum obscuration location and corresponding TEC evolution at $30 \mathrm{~min}$ time steps.

To quantify the TEC depletion/deviation with respect to the mean/median TEC level, the absolute TEC deviations are computed by subtracting the 27 day median TEC values from the TEC values observed during the eclipse time. Figure 4 shows absolute TEC deviation maps at the same time instances of $8: 38,9: 38,10: 08$, and 10:38 UT, as in Figure 3. The negative values indicate a TEC reduction of up to 6 TEC units at the European region during the eclipse. The subsequent plots show the location of maximum obscuration by filled black circles and the corresponding TEC depletion scenario at 30 min time steps. 
M.M. Hoque et al.: Ionospheric response over Europe during the solar eclipse of March 202015

(a)

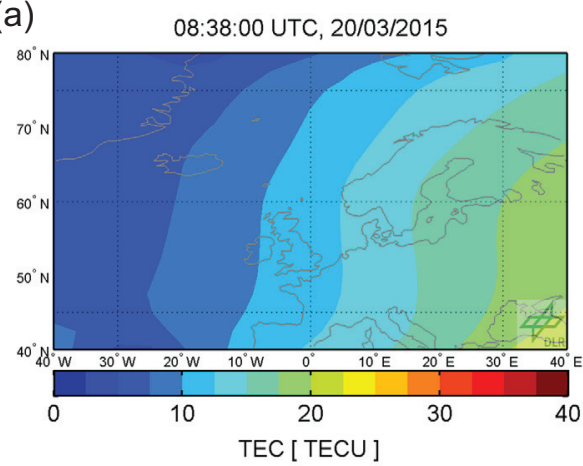

09:38:00 UTC, 20/03/2015

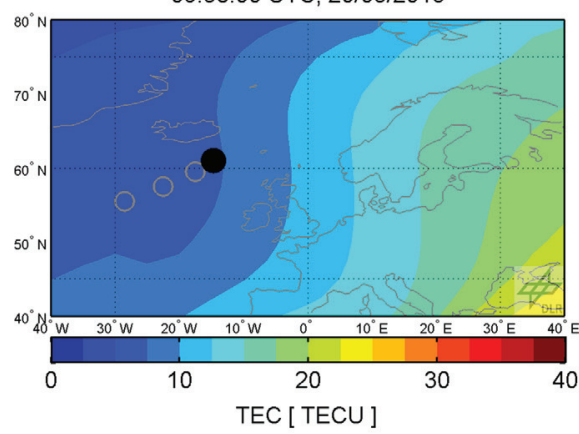

10:08:00 UTC, 20/03/2015

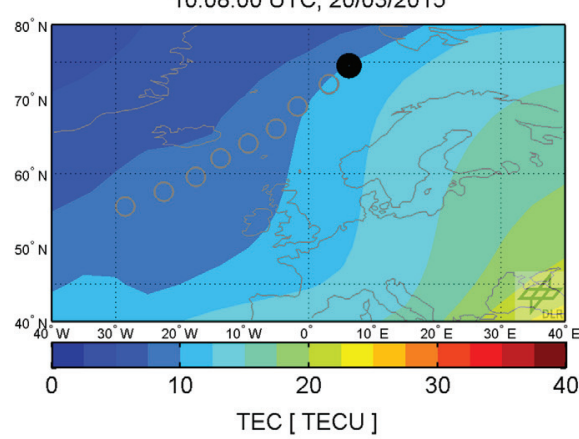

10:38:00 UTC, $20 / 03 / 2015$

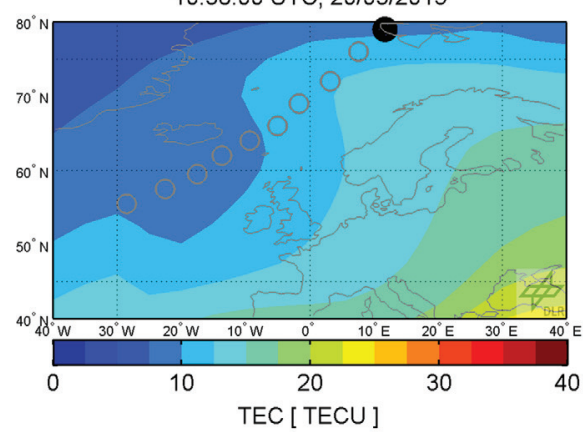

(b)

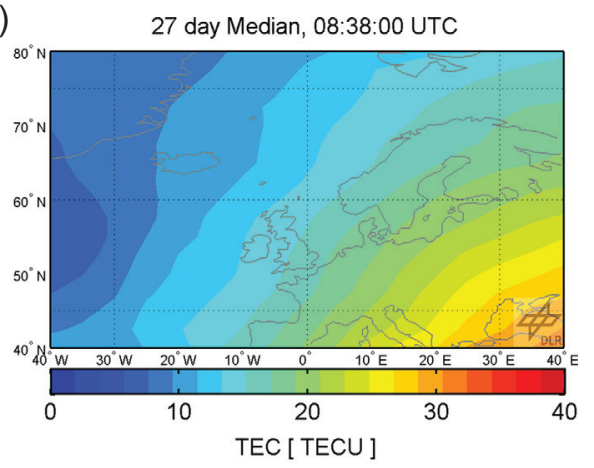

27 day Median, 09:38:00 UTC

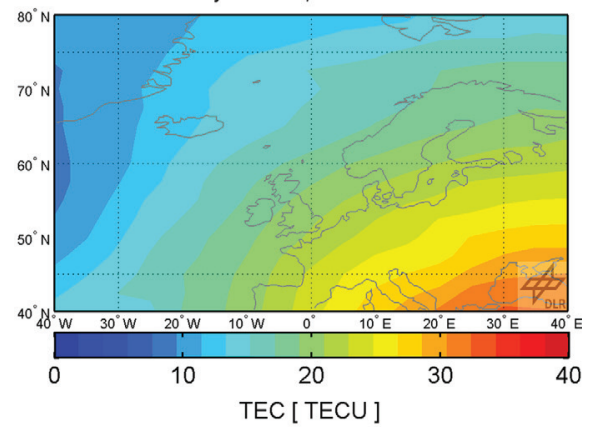

27 day Median, 10:08:00 UTC

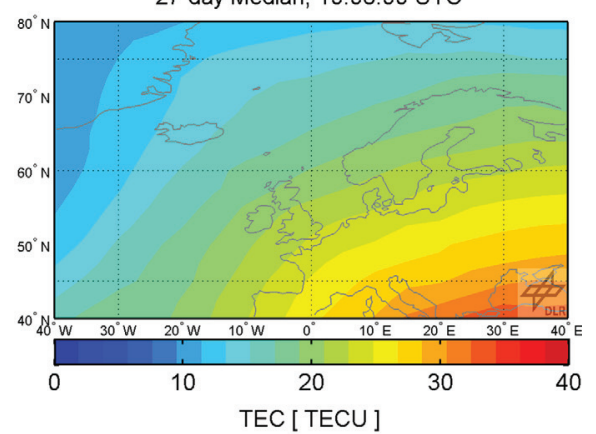

27 day Median, 10:38:00 UTC

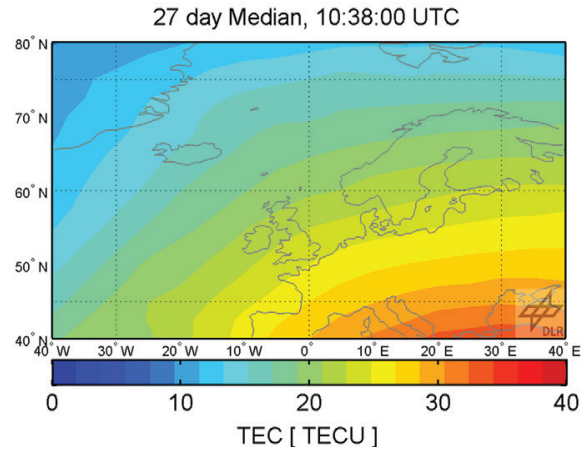

Fig. 3. (a) TEC maps over the European region $\left(40-80^{\circ} \mathrm{N}, 40^{\circ} \mathrm{W}-40^{\circ} \mathrm{E}\right)$ at $8: 38,9: 38,10: 08$, and 10:38 UT on solar eclipse day March 20 , 2015. (b) The corresponding 27 day median TEC maps.

We found that the median TEC at high latitudes hardly exceeded the 10 TEC unit level during morning hours when the solar eclipse occurred. This indicates large relative TEC deviations during the eclipse period with respect to the median values. To quantify the relative TEC depletion, we computed the percentage TEC deviation

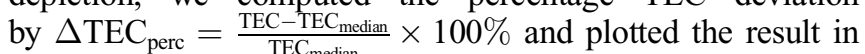
Figure 5. The $\mathrm{TEC}_{\text {median }}$ is computed from the TEC database of 27 days from doy 66 to 92 . Thus, at each grid point, we have 27 values for each time step and the median values are calculated separately at each grid point for each time step. Now, the ionospheric dynamics during the solar eclipse such as a large depletion zone around the shadow spot and its movement become obvious. In general, the depletion exceeds $50 \%$ inside the shadow. Comparing the subsequent maps between 08:38 and 10:38 UT, we find that the depletion zone extends far into the low latitudes, more precisely along the south-east direction. Such extension indicates that plasma transport processes are activated during the eclipse (Jakowski et al. 2008). The depletion persists even hours after 

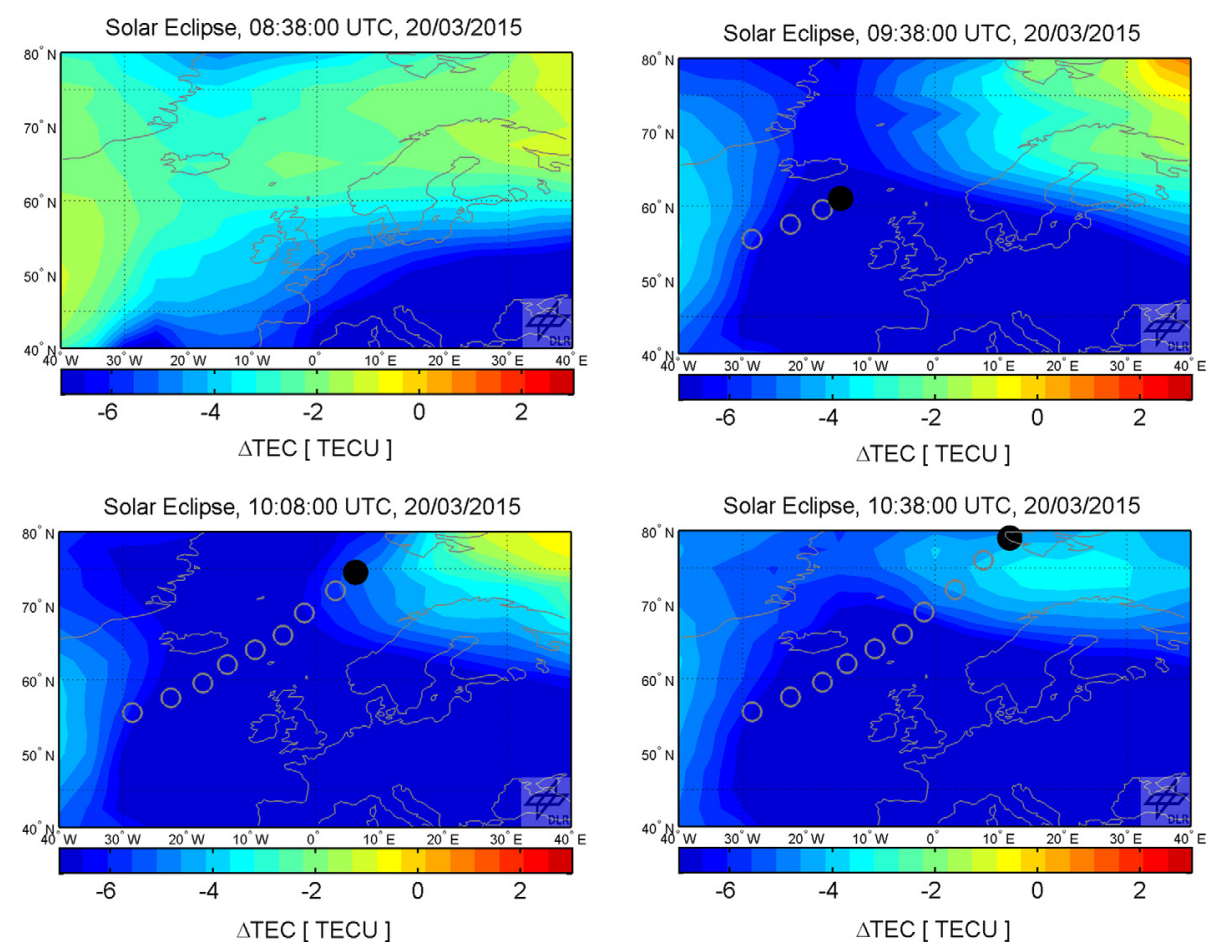

Fig. 4. The absolute TEC deviation from 27 day median values during solar eclipse.

(a)

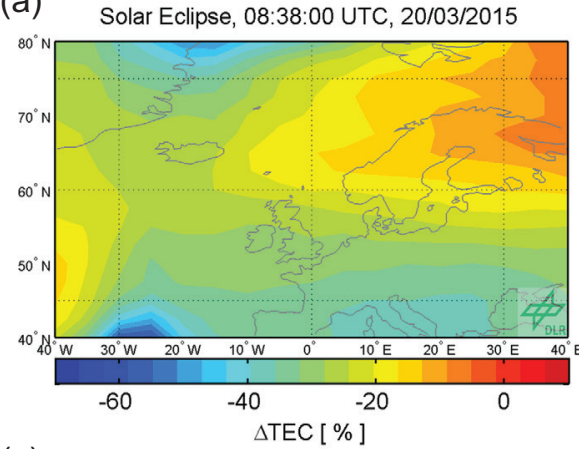

(c)

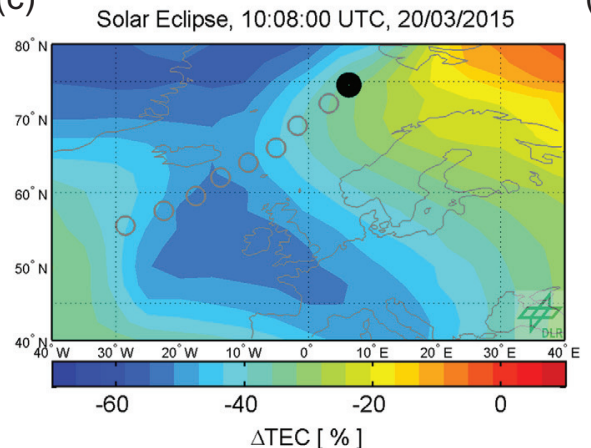

(b)

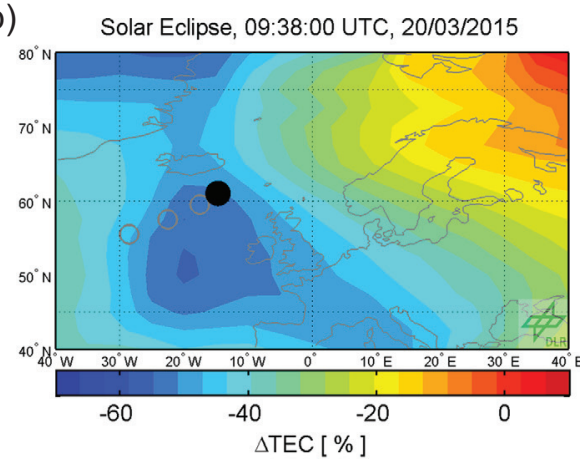

(d)

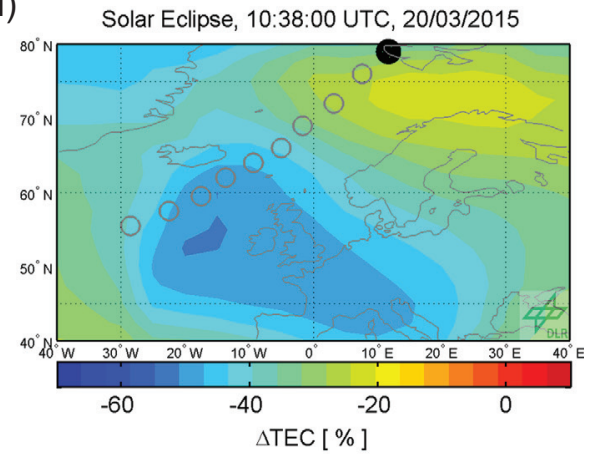

Fig. 5. Percentage TEC deviation with respect to 27 day median values during the solar eclipse.

the maximum obscuration, which indicates that the recovery of plasma density is decoupled from the obscuration function.

Investigating the large depletion zone around the shadow spot (Fig. 5), we found TEC reduction of up to 6 TEC units and total plasma depletion reached even over $50 \%$ (Fig. 5). The observed TEC depletion is higher than those reported earlier for the 1999 and 2005 eclipses by
Jakowski et al. (1999, 2008) and also others (Tsai \& Liu 1999; Rashid et al. 2006). Jakowski et al. (2008) found TEC reduction of up to several TEC units and the total plasma depletion of about $40 \%$ during the eclipse on August 11, 1999 and about 30\% during the eclipse on October 3, 2005. The unusual high depletion reported here is obviously due to the negative bias of up to $20 \%$ already observed over Northern 
M.M. Hoque et al.: Ionospheric response over Europe during the solar eclipse of March 202015

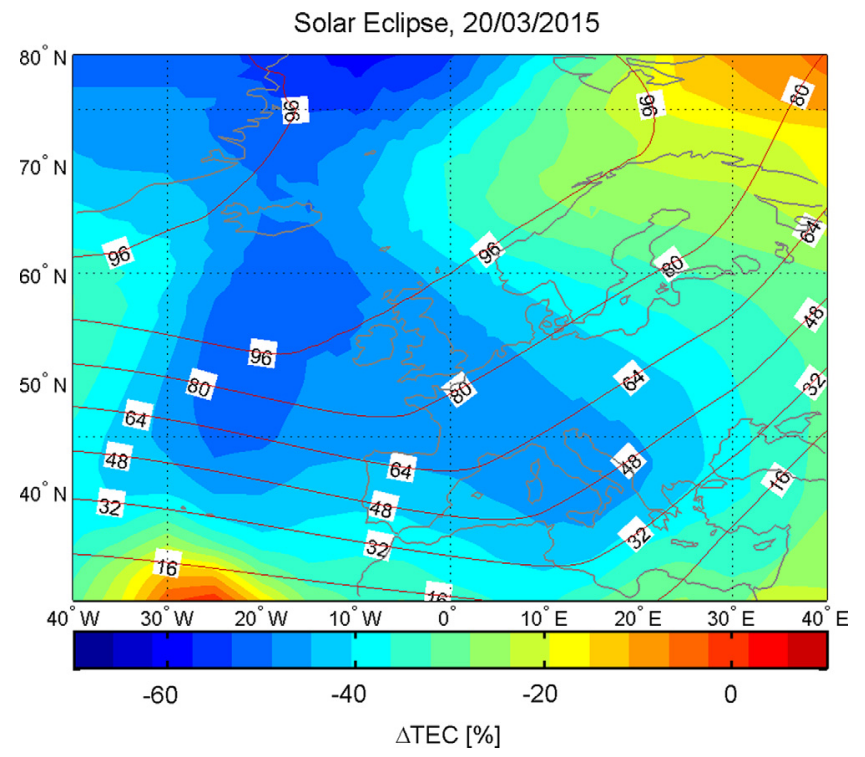

Fig. 6. Percentage TEC deviation when maximum obscuration occurs. The iso-lines show the maximum obscuration values.

Europe before the eclipse occurred (cf. Fig. 5a). As mentioned before, the March 20, 2015 eclipse occurred during the recovery phase of a strong geomagnetic storm that began three days before on March 17, 2015, and as a result, it is assumed that the ionosphere was still perturbed and depleted (negative storm phase).

The mean TEC level found of 5-10 TECU (see Fig. 3a) was much lower in 2015 at the large depletion zone around the shadow spot compared to years 1999 and 2005, in which the mean TEC level found was 25-30 and 15-20 TECU, respectively (Jakowski et al. 2008). We found a quite different eclipse path for 2015, compared to years 1999 and 2005. In 2015 , the eclipse path moved from low to high latitudes, whereas in 1999 and 2005 the eclipse paths moved the other way around, i.e., from comparatively high to low latitudes. Therefore, the large depletion zone around the shadow spot was observed at low and mid latitudes around $35-45^{\circ} \mathrm{N}$ in 2005 , whereas in 2015 , it was observed at high latitudes around $55-65^{\circ} \mathrm{N}$, providing lower mean TEC levels and consequently a higher percentage for TEC depletion. The depletion zone covers a large area up to low latitudes. It is also interesting that the eclipse shows different latitudinal depletion patterns over time, compared to the previous solar eclipses on August 11, 1999 and October 3, 2005. Whereas the depletion zone extends roughly horizontally from west to east in the 1999 and 2005 eclipses, the depletion zone extends toward lower latitudes during the eclipse of March 20, 2015. Considering eclipse geometry, on March 20, 2015, the obscuration maximum appears at higher latitudes later.

To investigate the spread of the TEC depletion region in relation to the maximum obscuration function, we plotted the maximum obscuration values over the percentage TEC deviation map in Figure 6. However, the figure does not map the TEC deviation for a specific time instance as before. Rather, it shows TEC deviation values corresponding to the time when the maximum obscuration occurred at each grid point over the solar eclipse period, i.e., 8-11:40 UT hours. The maximum obscuration values are plotted as lines of identical value (e.g., 96, 80, ...) also indicating the path of the solar eclipse over Europe. Figure 6 shows that the spread of the TEC

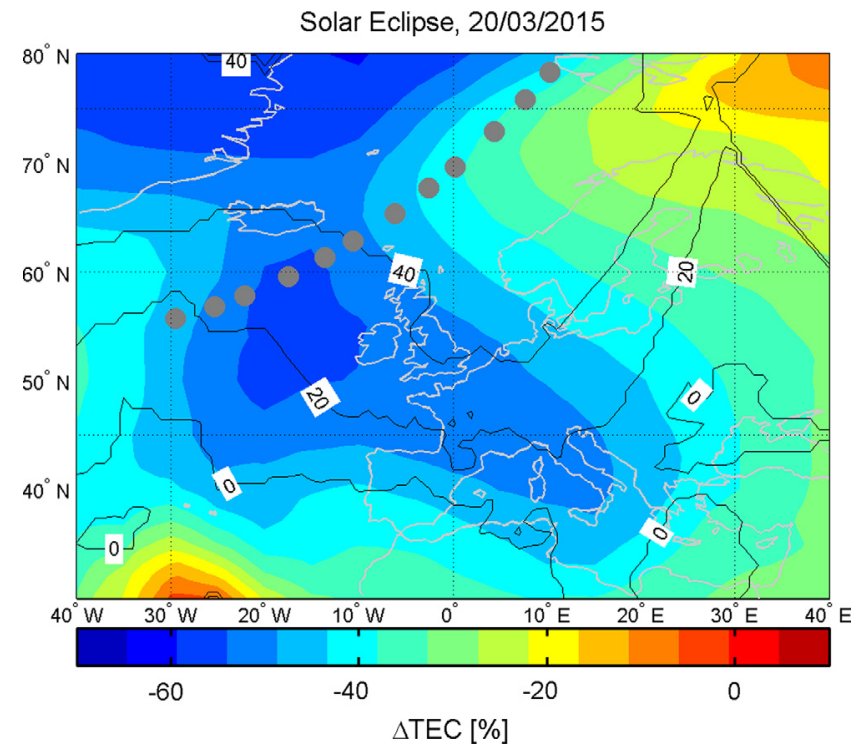

Fig. 7. Maximum percentage TEC deviation during the solar eclipse period. The iso-lines show the time difference in minutes between the maximum obscuration and maximum TEC deviation time instances.

depletion region roughly follows the structure/shape of the maximum obscuration curves.

Figure 7 shows a map of maximum percentage TEC deviation during the solar eclipse period. The maximum TEC deviations were observed over the European region at different times depending on the eclipse path and time of maximum obscuration at a certain location. The delay between the time of the maximum obscuration and the time of observed maximum TEC deviation is shown as iso-line plots over the TEC deviation map. The iso-line plots in Figure 7 show that the ionospheric response to the obscuration function is delayed up to $40 \mathrm{~min}$ in the vicinity of the totality zone. The shortest delay time of TEC response is found as 0 min approximately near and below $40^{\circ} \mathrm{N}$ latitudes. These results do not confirm an increasing delay of the minimum TEC level with the distance from the eclipse path as concluded by Krankowski et al. (2008).

However, our present observation is in general agreement with earlier observations of 20-30 min delay found during the solar eclipse on October 3, 2005 (Jakowski et al. 2008; Krankowski et al. 2008) and other eclipses (e.g., Cohen 1984). TEC is an integral measure and delayed response is expected since the topside ionosphere is less prone to radiation and is rather influenced by plasma redistribution, while the bottomside ionosphere is mainly controlled by local solar radiation (Cheng et al. 1992). It can be seen that the delay practically vanishes when the maximum obscuration is about less than $30 \%$. This is probably due to the fact that the solar eclipse occurs in the morning hours, i.e., the increasing solar radiation should be able to compensate the delay by increasing photoionization.

As already mentioned, we reconstructed TEC maps at every $5 \mathrm{~min}$ interval. This allowed us to plot time series of TEC along selected meridians (e.g., $-5^{\circ} \mathrm{E}, 5^{\circ} \mathrm{E}, 15^{\circ} \mathrm{E}$, etc.). The subsequent plots in Figure 8 show how the TEC depletion develops along the meridian during the eclipse. The plots show a sudden drop in ionospheric TEC due to switching off (obscuration) of solar radiation. The difference 
(a)

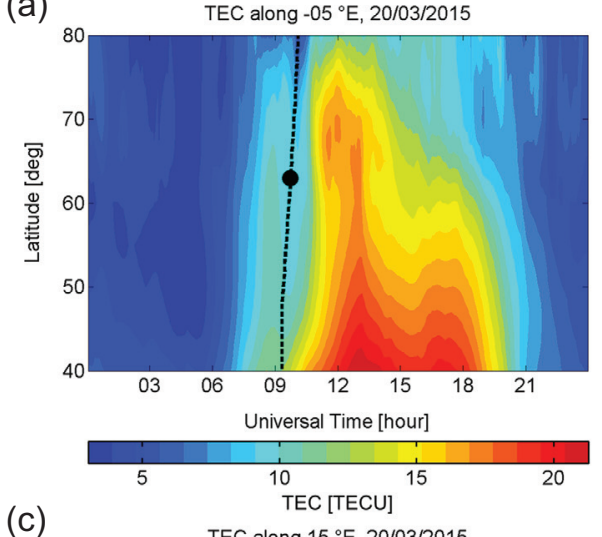

(c)

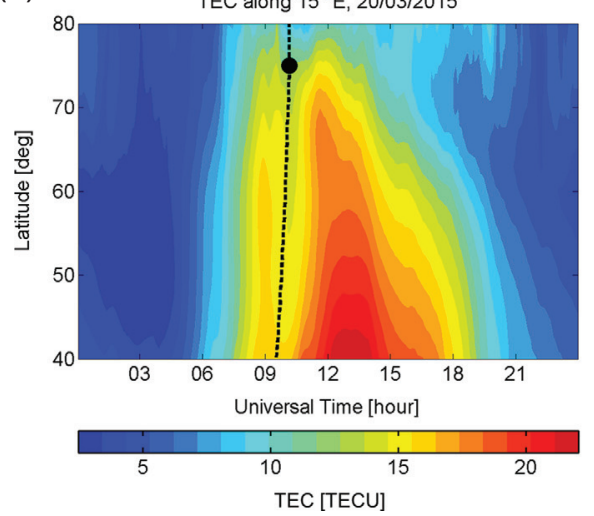

(b)

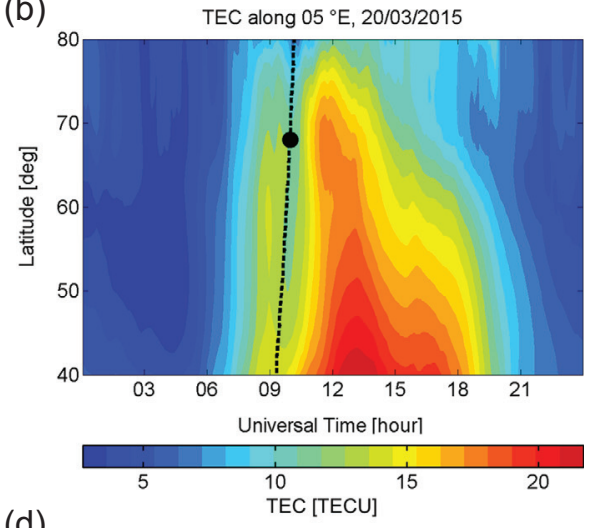

(d)

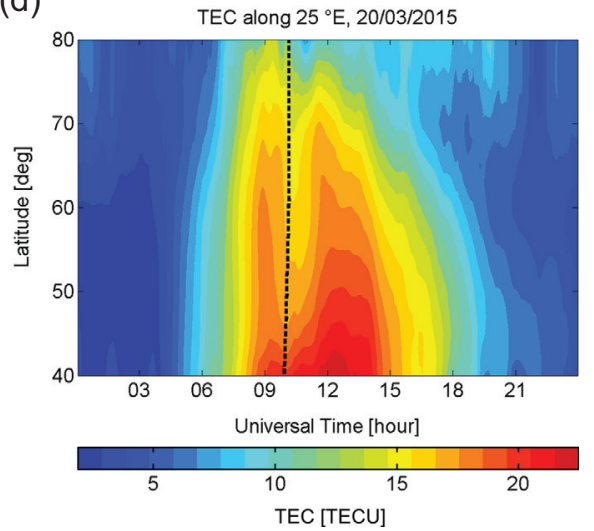

Fig. 8. Diurnal variation of total electron content along selected meridians on March 20, 2015. The black dotted line shows the obscuration path where the black circle gives the total eclipse location.

between different meridian maps may be explained by the different local times of the maximum obscuration. We found that typical diurnal TEC variation is perturbed, showing the presence of two peaks at $20^{\circ} \mathrm{E}$ or higher meridian (see Fig. 8d).

Instead of its integral nature, the TEC value can reflect wave-like phenomena such as TIDs. However, gravity waves are only visible if the wave amplitude is large enough and the ray path geometry does not cancel out the wave signatures in the integral (Davis \& Da Rosa 1970; Jakowski et al. 1983, 2001; Tsai \& Liu 1999; Rashid et al. 2006). Moreover, the geomagnetic activity influences the generation mechanism of these TIDs. We found that although the eclipse day shows a moderate $\mathrm{Kp}$ value (4.7), the previous days show higher $\mathrm{Kp}$ values (up to 7.7), indicating significant geomagnetic activity. Examining the GPS TEC data, we found evidence of wave activity especially at low-latitude stations during the solar eclipse. However, TIDs are frequently observed in the TEC data, demonstrating the natural variability of TEC. Since the wave activity observed in TEC at different European location on March 20, 2015 started earlier than the solar eclipse appeared over Europe, tropospheric sources should not be ignored when considering the origin of gravity waves (Jakowski et al. 2001). However, detailed investigation of TIDs is out of the scope of the current paper.

\subsection{Vertical sounding data}

As already discussed, the topside ionosphere is mainly characterized by plasma redistribution processes and therefore plasma content grows because diffusion processes need some time to come into effect. However, due to the presence of strong photoionization and recombination processes, the ionosphere at lower altitudes responds faster to local solar radiation, i.e., sudden changes in the obscuration function. Therefore, the VS measurements are highly sensitive to local effects as well as plasma redistribution processes, although they cannot provide accurate information about the total ionization loss in relation to the obscuration function during a solar eclipse.

The diurnal variations of ionospheric key parameters, such as the maximum plasma frequencies foF2, foF1, and foE at the ionospheric F2 $(\approx 200-400 \mathrm{~km}), \mathrm{F} 1(\approx 120-200 \mathrm{~km})$, and $\mathrm{E}$ $(\approx 90-120 \mathrm{~km})$ layers, respectively, and the F2 layer peak density height hmF2, are plotted in Figure 9 at ionosonde stations DB049 and EB040 during the solar eclipse. The corresponding peak electron density NmF2, GPS-derived vertical TEC and slab thickness values are plotted in Figure 10. The time interval, where the obscuration function is non-zero, is highlighted by a gray shadow.

Besides a strong depletion of the F2 layer in the course of the solar eclipse, Figure 9 also shows a clear F1 layer depletion. Due to the fast recombination processes at $\mathrm{E}$ and $\mathrm{F} 1$ layer heights, a similar variation of $\mathrm{E}$ and $\mathrm{F} 1$ layer electron densities is expected. Figure 9a indicates this shortly after the start of the eclipse when foE started to decrease gradually. The foE values dropped from about $2.8 \mathrm{MHz}$ at $08: 30$ UTC to $2.5 \mathrm{MHz}$ within $15 \mathrm{~min}$ and remained unchanged for about $1.5 \mathrm{~h}$ until about 10:45 UTC, when they again started to increase. Similar results were found by Verhulst et al. (2016) when analyzing data from the Dourbes station to investigate signatures of TIDs during the solar eclipse. They reported that 
M.M. Hoque et al.: Ionospheric response over Europe during the solar eclipse of March 202015
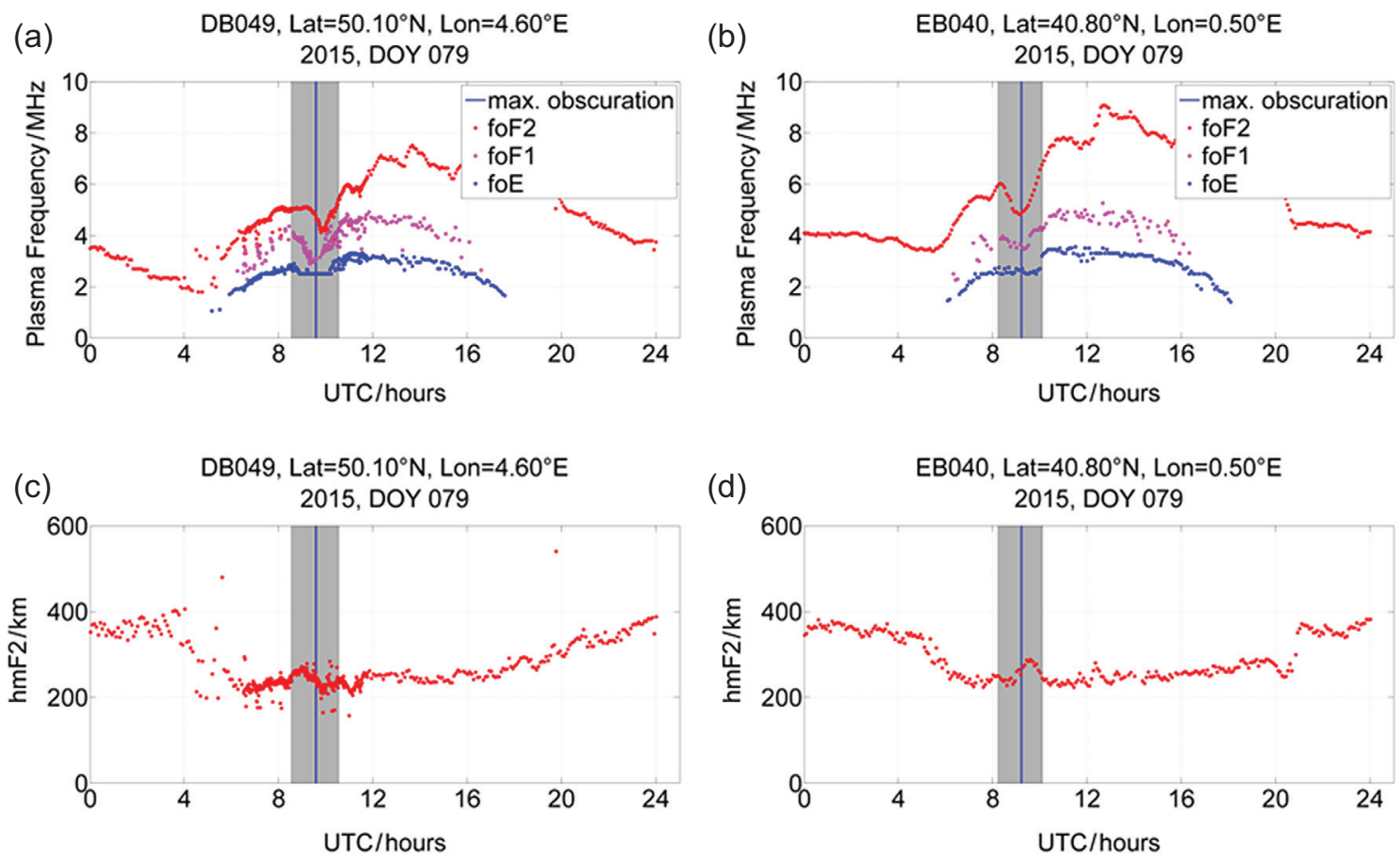

Fig. 9. Diurnal variation of plasma frequencies foF2, foF1, foE and peak height hmF2 at ionosonde stations DB049 and EB040 during the solar eclipse on March 20, 2015.
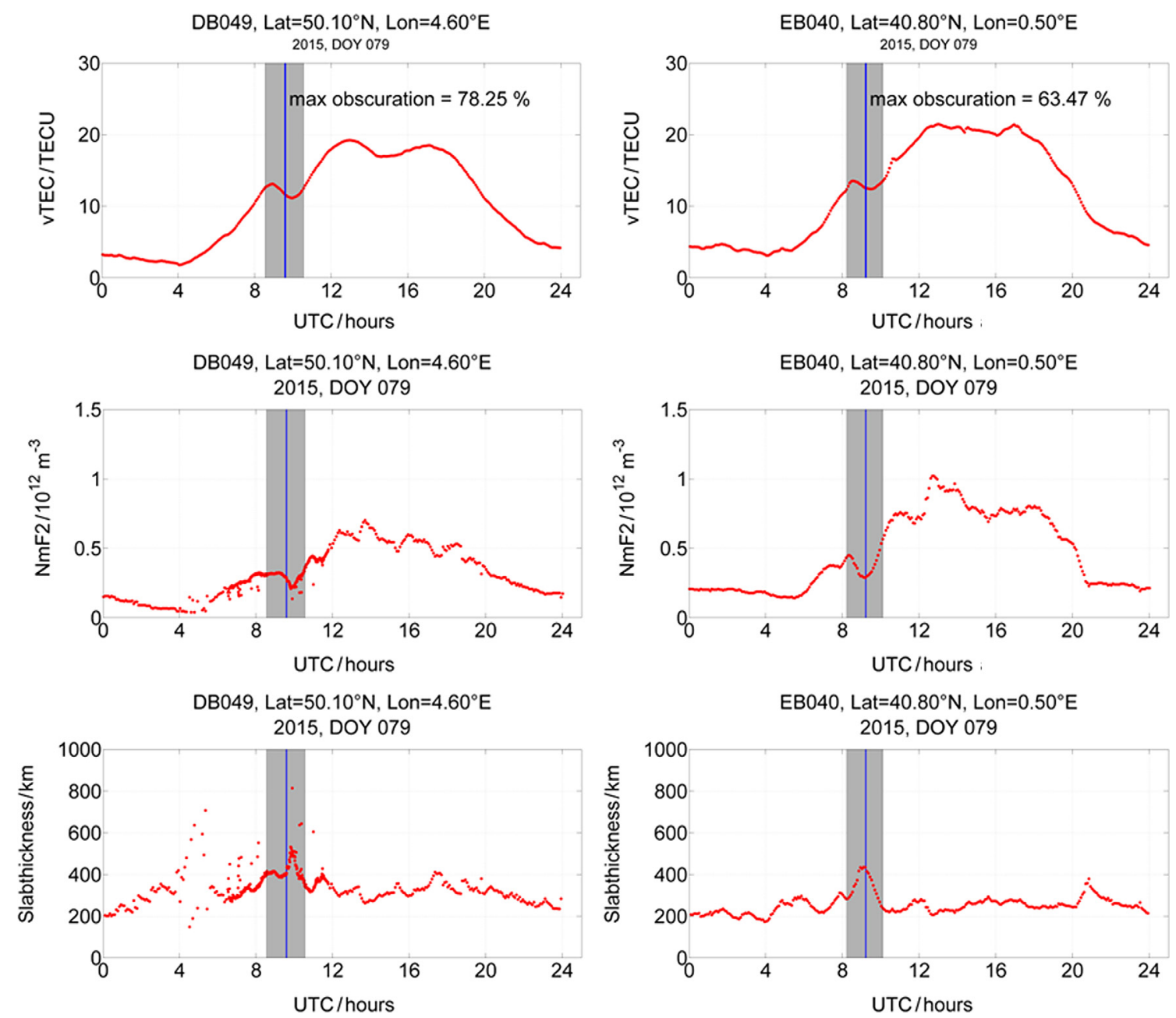

Fig. 10. Diurnal variation of TEC, NmF2, and slab thickness at DB049 and EB040 during the solar eclipse on March 20, 2015. 
although the Digisonde is equipped with the latest version of the automatic ionogram interpreter Artist-5 (Galkin \& Reinisch 2008), it had difficulties identifying a proper trace of the E layer and therefore provided a default daytime value of $2.5 \mathrm{MHz}$. As a result, it is reasonable to assume that the E layer was further depleted at around the time of the maximum obscuration at the Dourbes station.

Based on simulation studies, Le et al. (2008) discussed the eclipse effects on electron density and corresponding delay time considering control factors such as the background hmF2, local time and solar zenith angle, solar cycle, and dip angle. According to their study, an eclipse will produce a relatively smaller change in $\mathrm{NmF} 2$ associated with a higher time delay if the hmF2 is higher. Again, they found that the eclipse effect is larger at midday than in the morning and afternoon, and a larger dip angle results in a smaller eclipse effect. Figure 9a shows that the delay time of the ionization decrease increases with height from foE to foF2 in agreement with earlier observations (e.g., Jakowski et al. 2008) and simulation results (e.g. Le et al. 2008).

Since photoionization maximizes at foF1 altitudes, this layer reacts immediately in case of solar radiation flux changes. The situation is different at hmF2 and at the topside ionosphere where time-consuming redistribution processes have to be taken into account.

Considering the ionosonde station DB049, the time delay is about $13 \mathrm{~min}$ in $\mathrm{NmF} 2$ at $250 \mathrm{~km}$ height and about $20 \mathrm{~min}$ at about $350-400 \mathrm{~km}$ height as shown in TEC. Considering the ionosonde station EB040, there is practically no delay at hmF2 heights, whereas it is about $20 \mathrm{~min}$ in TEC (cf. Fig. 10). This is probably due to the increasing photoionization rate in the morning hours that is stronger at lower latitudes compared with the Dourbes station. Thus, also taking into account the lower obscuration over EB040, the increasing photoionization rate may compensate for the solar eclipseinduced loss rate in the bottomside ionosphere, leading to a zero delay at $\mathrm{hmF} 2$ and below. This explanation is only valid if the background ionosphere behaves regularly, i.e., external forces of non-eclipse origin can be ignored. In other cases, the delay times may range up to $120 \mathrm{~min}$ as reported by Tsai \& Liu (1999) when studying the solar eclipses on October 24, 1995 and March 9, 1997 at low latitudes. Furthermore, Afraimovich et al. (2002) reported very short response times of only a few minutes. Thus, to explain the delay times correctly, one has to take into account the coupled thermosphere-ionosphere system. The low-latitude ionosphere is strongly influenced by dynamic processes related to the equatorial anomaly where the transport processes dominate the photochemical processes.

At both ionosonde stations, wave-like processes are indicated which superpose to the eclipse bite-out ionization. The examination of the vertical TEC data with respect to the increased wave activity during the eclipse over ionosonde stations DB049 and EB040 hardly produced evidence of such activity. Due to the integral nature of TEC, it reflects wave-like phenomena in a different way than what the VS observation does. Wave activities are still visible in the TEC data if the wave amplitude is large enough and the ray path geometry does not cancel out the wave signatures in the integral measure of TEC.

As mentioned before, the combination of $\mathrm{NmF} 2$ and TEC over the ionosonde stations enables us to compute the equivalent slab thickness $\tau$. The profile shape variation during the eclipse is reflected in the equivalent slab thickness $\tau$ by $\tau=\mathrm{TEC} / \mathrm{NmF} 2$, which is closely related to the neutral gas scale height under diffusive equilibrium conditions. Since the time delay between F2 layer height and topside ionosphere is different, the ratio increases considerably when $\mathrm{NmF} 2$ reaches a minimum, whereas TEC has not yet reached the minimum.

Indeed, Figure 10 shows a strong increase of slab thickness values by about $80-100 \mathrm{~km}$ when solar radiation is reduced during the eclipse. The enhanced slab thickness shows evidence for a delayed depletion of the topside ionosphere. After the maximum obscuration, the slab thickness decreased rapidly. It should be noted that the significant enhancement of the slab thickness is in contradiction with the cooling of the thermosphere. Under diffusive equilibrium conditions, the slab thickness is proportional to the neutral gas scale height, i.e., proportional to the temperature. The rapid decrease of the slab thickness after reaching the peak value is probably due to the thermospheric cooling effect that is also delayed with respect to the obscuration function. Figure 11 shows the percentage deviations of $\mathrm{NmF} 2$ and slab thickness with respect to their 27 day median values on the solar eclipse day. We find at both stations that the slab thickness deviations of about $60-80 \%$ are much higher than the $\mathrm{NmF} 2$ deviations of about $20-25 \%$ from the background level when the obscuration occurs at both stations (see gray shaded area). This observation again indicates evidence for a delayed response of the topside ionosphere reflected in the TEC integral.

\subsection{VLF propagation}

Since VLF signals are reflected back at the lower ionosphere more specifically at the D layer, we expect faster response in signal strength changes during the eclipse event. Figure 12 shows diurnal variations of VLF signal strength received in the NZ station from 6 Navy stations between frequencies 20 and $100 \mathrm{kHz}$ on the eclipse day. Different propagation paths suffer different levels of obscuration and the duration of obscuration for each transmitter-receiver path is marked in gray shadow. The signals received from different Navy stations show a clear and immediate variation of the signal strength fluctuation during the eclipse period. We found maximum fluctuations for stations NRK, GXH, GYN, FTA63, FUG, and TBB as $6.66,7.97,6.55,1.61,3.98$, and $1.16 \mathrm{~dB}$, respectively (see Table 1). However, since the transmission frequency and power are different for each Navy station, a straightforward comparison among the amplitudes of the signal strength fluctuation during solar eclipse is not justified. It is interesting to compare the delay in response time for different links with respect to the delay found in the GPS TEC. For this purpose, the relative VLF amplitudes are plotted in Figure 13a after levelling unperturbed amplitudes to zero together with TEC fluctuations at mid-reflection points (Fig. 13b). The time of the maximum obscuration is marked by a black vertical line in each subplot. Examining the peak signal fluctuation, we found immediate response to the maximum obscuration in case of signals received from the NRK, GXH, GYN, and TBB stations, whereas we observed about 5-15 min delayed depletion in TEC over these stations. We found wave-like signatures in the GPS TEC during the eclipse day over the NRK and GXH stations. However, the peak fluctuation shows a delayed response of about 6-12 min to maximum obscuration for signals received from the FTA and FUG stations. TEC over these stations shows maximum depletion delay of about 15-20 min. The TBB station is located farthest from the 
M.M. Hoque et al.: Ionospheric response over Europe during the solar eclipse of March 202015
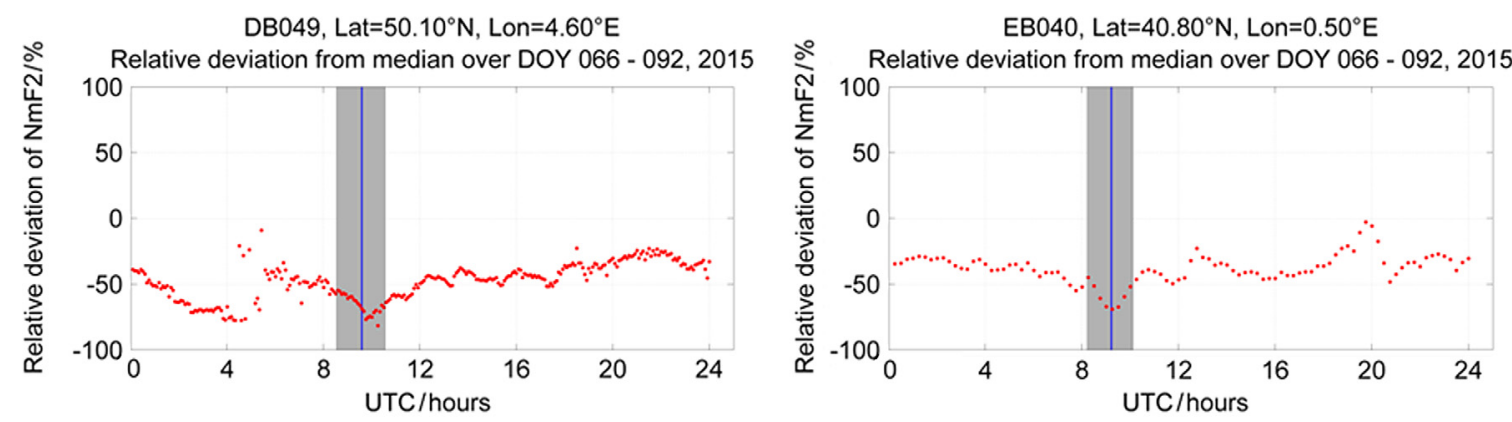

DB049, Lat $=50.10^{\circ} \mathrm{N}$, Lon $=4.60^{\circ} \mathrm{E}$

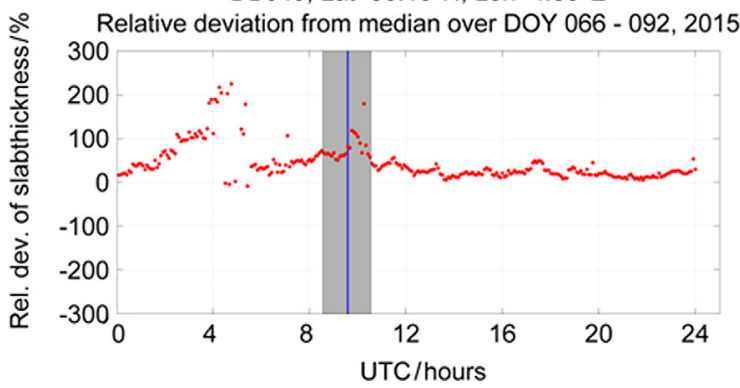

EB0 40, Lat $=40.80^{\circ} \mathrm{N}$, Lon $=0.50^{\circ} \mathrm{E}$

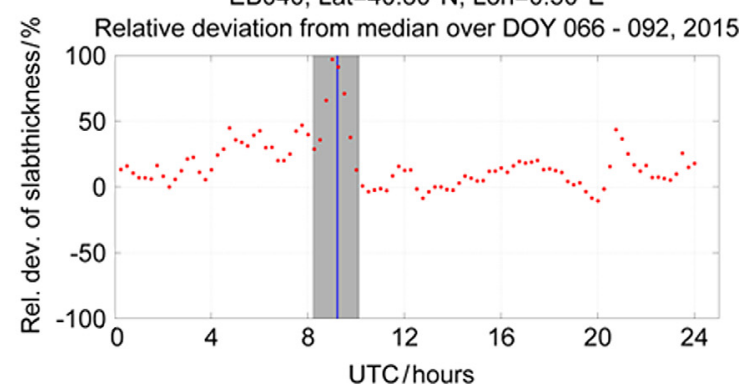

Fig. 11. Relative deviations of NmF2 and slab thickness for the March 20, 2015 solar eclipse.

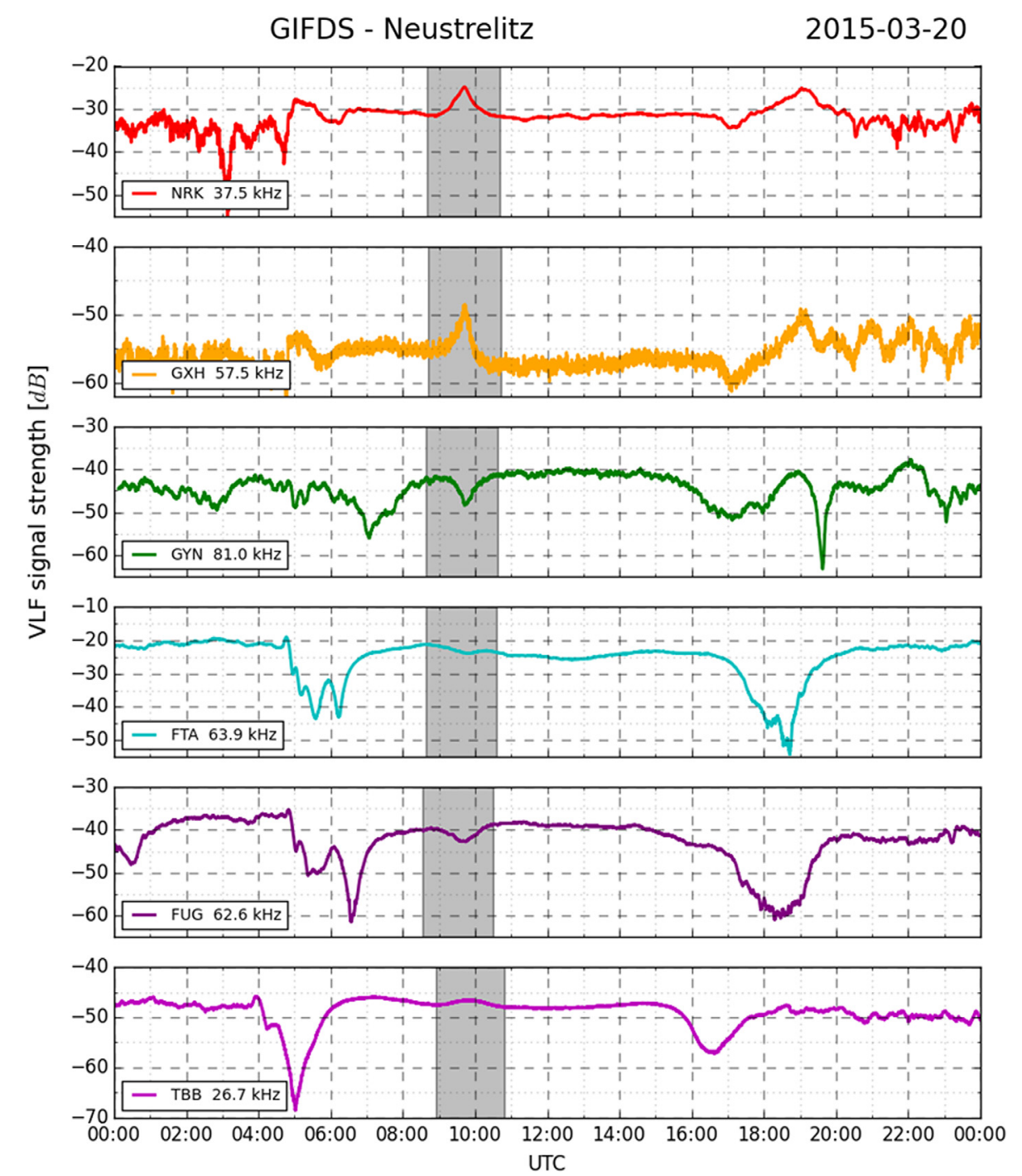

Fig. 12. Diurnal variations of VLF signal strength received in the NZ station from 6 Navy stations between frequencies of 20 and $100 \mathrm{kHz}$ on the eclipse day March 20, 2015. 
J. Space Weather Space Clim., 6, A36 (2016)

(a)
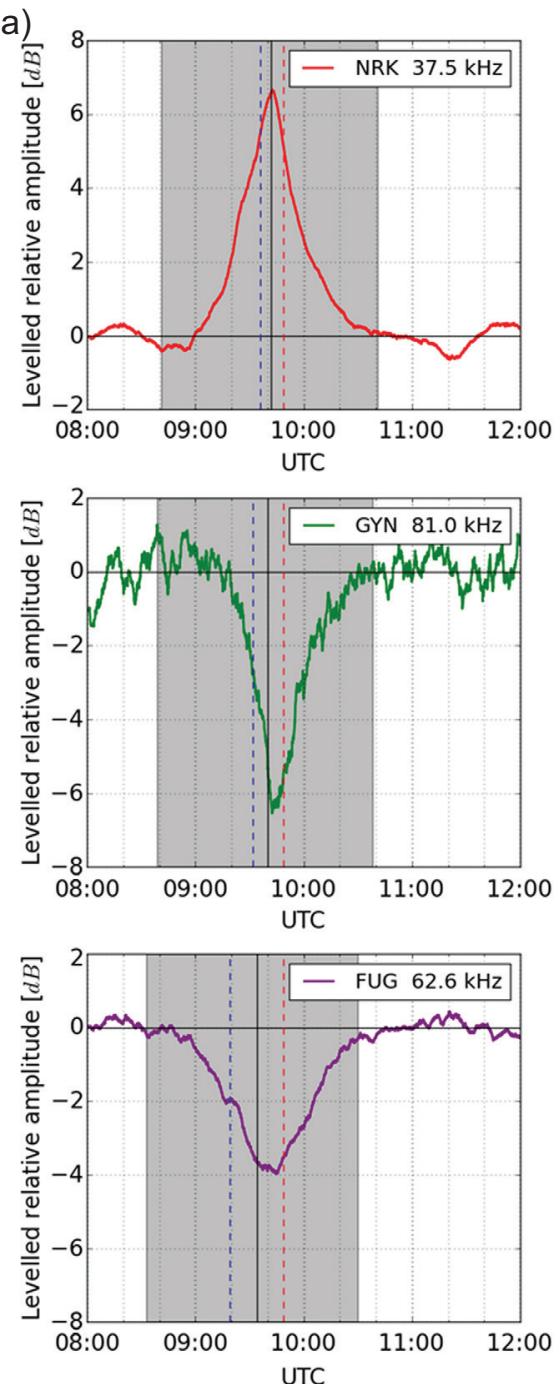

(b)
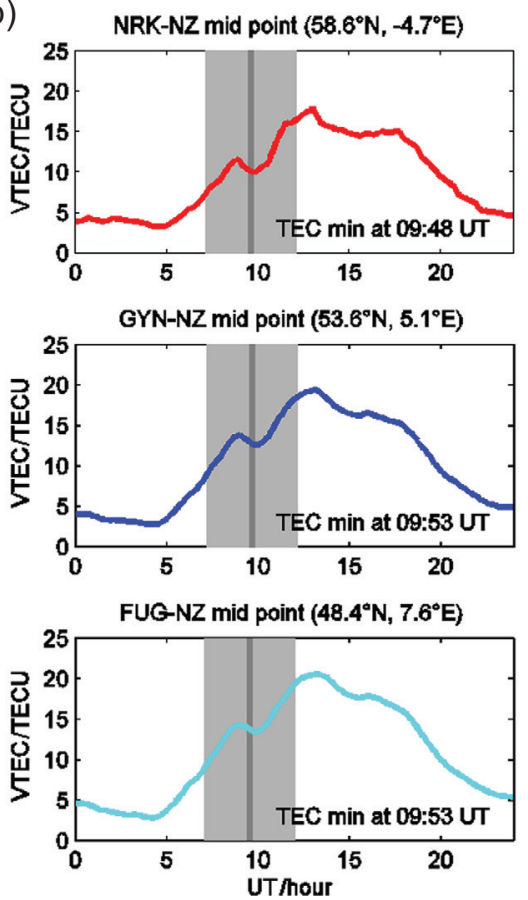
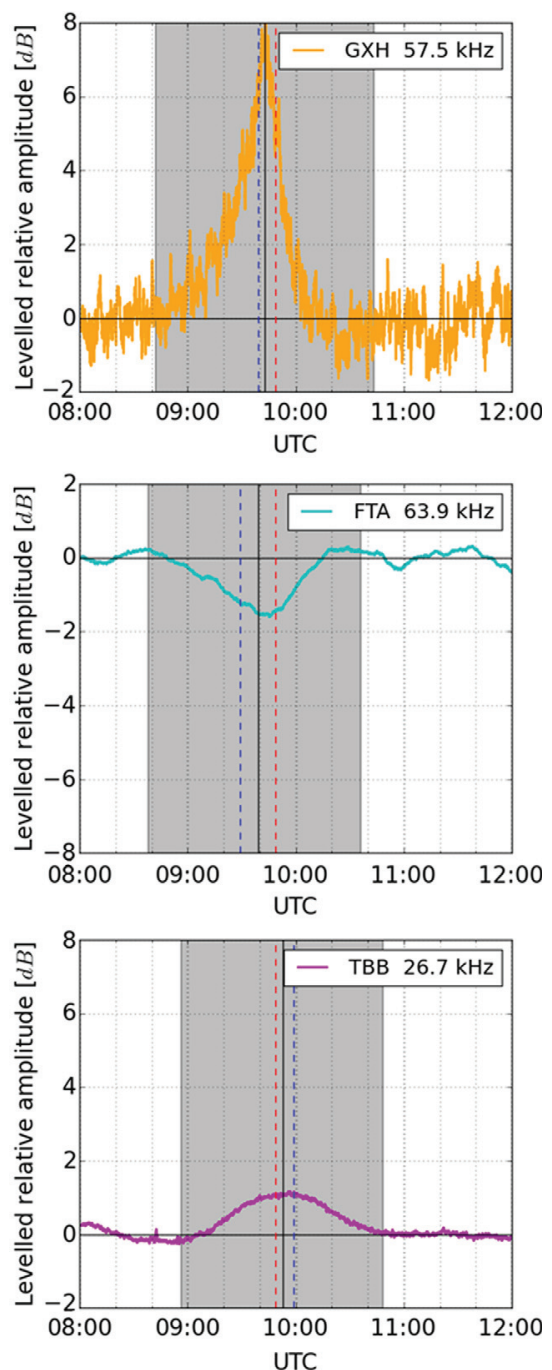

GXH-NZ mid point $\left(56^{\circ} \mathrm{N}, 4.7^{\circ} \mathrm{E}\right)$

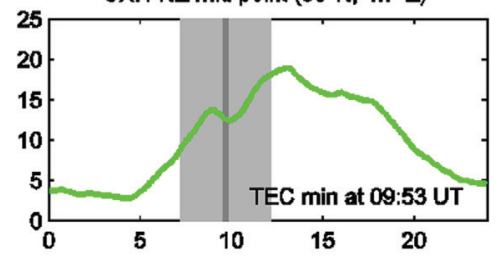

FTA-NZ mid point $\left(50.9^{\circ} \mathrm{N}, 7.8^{\circ} \mathrm{E}\right)$
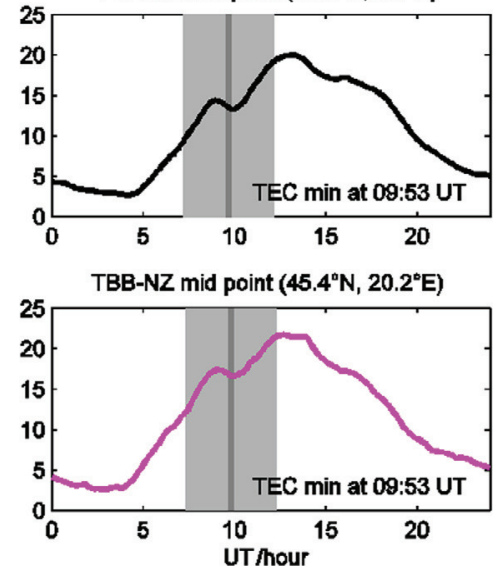

Fig. 13. (a) The relative fluctuation of VLF signal strength after levelling unperturbed amplitudes to zero. (b) TEC fluctuations at mid-reflection points are plotted. 
M.M. Hoque et al.: Ionospheric response over Europe during the solar eclipse of March 202015
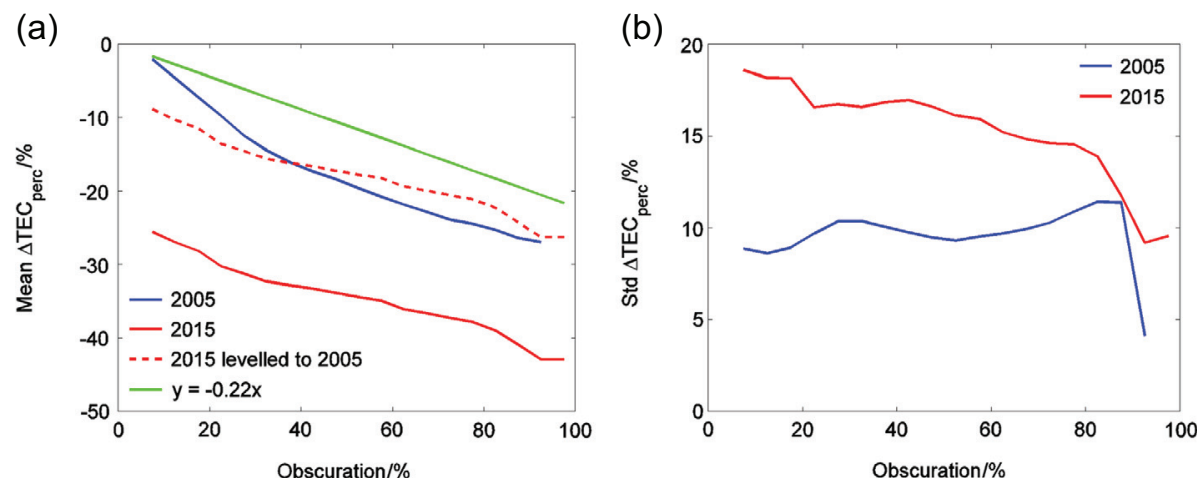

Fig. 14. (a) The mean and (b) standard deviation of $\Delta \mathrm{TEC}_{\text {perc }}$ as a function of obscuration during the solar eclipses on March 20,2015 and October 3, 2005.

eclipse path and is least affected by the obscuration. Similarly, the recovery of plasma density in the D layer is much faster and almost instantaneous with the increasing solar illumination, whereas TEC depletion still persists for hours.

\section{Possibility of modelling TEC depletion during solar eclipses}

For the following analysis, we computed gridded (latitude by longitude) maps of obscuration values for 2 min time steps during the solar eclipse on March 20, 2015. The data having obscuration values $<5 \%$ were discarded and the obscuration values were then sorted into bins of 5\% each (e.g., 5:5:100). Thus, each bin includes data from various latitude-longitude grid points and time instances. The corresponding $\Delta \mathrm{TEC}_{\mathrm{perc}}=\frac{\mathrm{TEC}^{\mathrm{TEC}} \text { median }}{\mathrm{TEC}} \times 100 \%$ (i.e., percentage deviation from 27 day median) values are taken into account separately for each bin to compute statistical estimates, such as means and standard deviations. Their values are plotted (see red colored plots) in Figure 14 as a function of the obscuration values.

It is interesting to note that we found a linear trend when the mean values of $\triangle \mathrm{TEC}_{\text {perc }}$ were plotted as a function of the obscuration. To verify whether the trend is valid for previous solar eclipse results, we computed $\triangle \mathrm{TEC}_{\text {perc }}$ and obscuration maps for the solar eclipse on October 3, 2005. Again the obscuration values are sorted into bins and corresponding $\triangle \mathrm{TEC}_{\mathrm{perc}}$ values are considered to estimate the mean and standard deviation for each bin. The estimated mean and standard values are plotted in Figure 14 (see blue colored plot). Again, we found a clear linear trend for the mean $\triangle \mathrm{TEC}_{\text {perc }}$ versus obscuration plot for the year 2005 . As Figure 14 shows, the two mean $\triangle \mathrm{TEC}_{\text {perc }}$ versus obscuration curves are approximately parallel, i.e., their slopes are approximately the same. However, when the obscuration started, we found high initial depletion/bias for the 2015 plot compared to the 2005 plot. The unusual high depletion might be due to the negative bias of up to $20 \%$ already observed over Northern Europe before the eclipse occurred (cf. Fig. 5a). When removing the bias of about $-20 \%$, the linear function of March 20 is very similar to the function obtained for the 2005 eclipse (see the dotted red colored plot). The linear dependency of the mean depletion on the obscuration independent of the solar eclipse indicates a possibility of modelling mean depletion as a function of obscuration.
Therefore, we derived a linear function, i.e., $y=-0.22 x$ combining data from both the depletion versus obscuration plots. This function provides an approximate estimate of relative TEC depletions as a function of obscuration values (see green colored plot) ignoring the initial depletion condition, which needs to be taken into account separately.

However, the standard deviation plots show high values above $10 \%$ and $15 \%$ during 2005 and 2015, respectively. The solar activity level indicated by the F10.7 shows 74.4 and 88.3 sfu for October 3, 2005 and March 20, 2015, respectively. The corresponding 27 day mean values were found to be 79.5 and $124.5 \mathrm{sfu}$, respectively. Comparing the two standard deviation plots, we found that the increase of the standard deviation values correlates with the increase of the solar activity level. Moreover, as already mentioned, the March 20, 2015 eclipse occurred during the recovery phase of a strong geomagnetic storm that began on March 17, 2015. The ionosphere was still depleted and this may contribute to high standard deviation values. Thus, in case of modelling the depletion, the preconditions of the background ionosphere have to be taken into account.

As we discussed, the initial depletion, location, solar incidence angle, and different local times of the maximum obscuration influence the level of plasma depletion. In the present paper, we only show the possibility of modelling the TEC response during a solar eclipse. Therefore, for simplicity we have not considered the solar incidence and local time effect when we sorted relative depletion. However, the high standard deviations obtained in the present analysis may be reduced when considering the initial depletion, delay in response time, solar incidence, and local time effect. This requires explicit modelling of TEC depletion during solar eclipses, which needs a comprehensive study considering different geophysical conditions. This is the subject of a future study.

As already mentioned, GNSS users can benefit from TEC depletion modelling during a solar eclipse. If the TEC depletion can be predicted in advance from such modelling activities, GNSS operators can either improve their broadcast delay/TEC information or inform users about the TEC depletion estimate depending on their location with respect to the eclipse path. Here we found up to 6 TECU depletion in the vertical TEC estimate around the shadow spot, which can be 2-3 times higher in slant TEC estimates at low elevation angles, indicating range errors of up to $2-3 \mathrm{~m}$ in singlefrequency GNSS positioning. 


\section{Conclusions}

The ionospheric response during the solar eclipse on March 20, 2015 was investigated using TEC, vertical sounding, and VLF signal strength measurements. Our TEC estimation shows that the total ionization reduces up to $50 \%$ (after bias correction $30 \%$ ) as a function of obscuration. Since this eclipse occurred during the negative phase of a severe geomagnetic storm on March 17, 2015, the observed TEC depletion was higher than those reported earlier for the 1999 and 2005 eclipses. Thus, a negative bias of up to $20 \%$ was already observed over Northern Europe before the eclipse occurred. Moreover, the eclipse path is quite different for the solar eclipse in 2015, compared to years 1999 and 2005. The large depletion zone around the shadow spot was observed at low and mid latitudes around $35-45^{\circ} \mathrm{N}$ in 2005 , whereas in 2015 , it was observed at high latitudes around $55-65^{\circ} \mathrm{N}$, providing lower mean TEC levels and consequently a higher percentage for TEC depletion. Nevertheless, it seems that the percentage TEC depletion depends linearly on solar obscuration nearly independently from actual solar eclipse conditions. The significant fluctuations in the VS measurements over the Ebro and Dourbes ionosonde stations indicate some wave activity that is not considered in detail because it seems that they have another origin.

The ionospheric response to the obscuration function is delayed up to $40 \mathrm{~min}$, decreasing with growing distance from the totality zone, and increasing with altitude. The increasing delay with altitude is in agreement with earlier findings for other eclipses. The equivalent slab thickness was found to increase by approximately $80-100 \mathrm{~km}$ during the solar eclipse in question. The enhanced slab thickness shows evidence for a delayed depletion of the topside ionosphere, assuming a regular background ionosphere. VLF measurements instead indicate an almost immediate response within a few minutes.

The findings of linear dependence of the percentage TEC depletion on solar obscuration during the eclipses on March 20, 2015 and October 3, 2005 need further investigation but indicate the possibility of modelling the TEC depletion as a function of the obscuration function during the solar eclipse under consideration. It is expected that GNSS users could reduce ionospheric propagation errors during a solar eclipse by applying such a model in single-frequency navigation and positioning.

Acknowledgements. We would like to thank the sponsors and operators of NASA's Earth Science Data Systems and the CDDIS for archiving and distributing the IGS data. We would like to acknowledge the support of the organizations contributing to the IGS by providing GNSS data to the CDDIS for the international scientific community. Thanks are also due to NOAA's NGDC for disseminating historical solar and magnetic data via SPIDR. We also thank SOPAC service for making daily GNSS satellite ephemeris data available. The editor thanks Ljiljana R. Cander and Huijun Le for their assistance in evaluating this paper.

\section{References}

Afraimovich, E.L., E.A. Kosogorov, and O.S. Lesyuta. Effects of the August 11, 1999 total solar eclipse as deduced from total electron content measurements at the GPS network. J. Atmos. Sol. Terr. Phys., 64, 1933-1941, 2002.

Altadill, D., J.G. Sole, and E.M. Apostolov. Vertical structure of a gravity wave like oscillation in the ionosphere generated by the solar eclipse of August 11, 1999. J. Geophys. Res., 106, 21419-21428, 2001.
Cheng, K., Y.N. Huang, and S.W. Chen. Ionospheric effects of the solar eclipse of September 23, 1987, around the equatorial anomaly crest region. J. Geophys. Res., 97, 103-111, 1992.

Chimonas, G., and C.O. Hines. Atmospheric gravity waves induced by a solar eclipse. J. Geophys. Res., 75, 875, 1970.

Clilverd, M.A., C.J. Rodger, N.R. Thomson, J. Lichtenberger, P. Steinbach, P. Cannon, and M.J. Angling. Total solar eclipse effects on VLF signals and modeling Observations. Radio Sci. 36, 773-788, 2001.

Cohen, E.A. The study of the effect of solar eclipses on the ionosphere based on satellite beacon observations. Radio Sci., 19 (3), 769-777, 1984.

Davis, M.J., and A.V. Da Rosa. Possible detection of atmospheric gravity waves generated by the solar eclipse. Nature, 226, 1123 , 1970.

Dow, J.M., R.E. Neilan, and C. Rizos. The International GNSS Service in a changing landscape of Global Navigation Satellite Systems. J. Geod., 83, 191-198, 2009, DOI: $10.1007 / \mathrm{s} 00190-008-0300-3$.

Fritts, D.C., and Z. Luo. Gravity wave forcing in the middle atmosphere due to reduced ozone heating during a solar eclipse. J. Geophys. Res., 98 (D2), 3011-3021, 1993.

Galkin, I.A., and B.W. Reinisch. The new Artist-5 for all digisondes. Ionosonde Network Advisory Group INAG Bulletin, 69, 1-8, 2008.

Hoque, M.M., and N. Jakowski. An alternative ionospheric correction model for global navigation satellite systems. J. Geod., 89, 391-406, 2014, DOI: 10.1007/s00190-014-0783-z.

Jakowski, N., H.D. Bettac, B. Lazo, L. Palacio, and L. Lois. The ionospheric response to the solar eclipse of 26 February 1979 observed in Havana/Cuba. Phys. Solariterr, 20, 110-116, 1983.

Jakowski, N., S. Schlueter, S. Heise, and J. Feltens. Satellite technology glimpses ionospheric response to solar eclipse. EOS, Transactions. American Geophysical Union, 80 (51), 621-626, 1999.

Jakowski, N., S. Heise, A. Wehrenpfennig, and S. Schlueter. Total electron content studies of the solar eclipse on 11 August 1999. CD-ROM, Proc. IBSS, Boston, MA4-6 June 2001, 279-283, 2001.

Jakowski, N., S. Stankov, V. Wilken, C. Borries, D. Altadill, et al. Ionospheric behaviour over Europe during the solar eclipse of 3 October 2005. J. Atmos. Sol. Terr. Phys., 70, 836-853, 2008.

Jakowski, N., C. Mayer, M.M. Hoque, and V. Wilken. TEC Models and their use in ionosphere monitoring. Radio Sci., 46, RS0D18, 2011, DOI: $10.1029 / 2010$ RS004620.

Krankowski, A., I.I. Shagimuratov, L.W. Baran, and G.A Yakimova. The effect of total solar eclipse of October 3, 2005, on the total electron content over Europe. Adv. Space Res., 41 (4), 628-638, 2008.

Le, H., L. Liu, X. Yue, and W. Wan. The mid-latitude F2 layer during solar eclipses: observations and modeling. J. Geophys. Res, 113, A08309, 2008, DOI: 10.1029/2007JA013012.

Le, H., L. Liu, X. Yue, W. Wan, and B. Ning. Latitudinal dependence of the ionospheric response to solar eclipse. J. Geophys. Res., 114, A07308, 2009, DOI: $10.1029 / 2009 J A 014072$.

Le, H., L. Liu, F. Ding, Z. Ren, Y. Chen, et al., Observations and modeling of the ionospheric behaviors over the east Asia zone during the 22 July 2009 solar eclipse, J. Geophys. Res., 115, A10313, 2010, DOI: 10.1029/2010JA015609.

Liu, J.Y., C.C. Hsiao, L.C. Tsai, C.H. Liu, F.S. Kuo, H.Y. Lue, and C.M. Huang. Vertical phase and group velocities of internal gravity waves derived from ionograms during the solar eclipse of 24 October 1995. J. Atmos. Sol. Terr. Phys., 60, 1679-1686, 1998.

Mueller-Wodarg, I.C.F., A.D. Aylward, and M. Lockwood. Effects of a mid-latitude solar eclipse on the thermosphere and ionosphere: a modelling study. Geophys. Res. Lett., 25, 3787-3790, 1998. 
M.M. Hoque et al.: Ionospheric response over Europe during the solar eclipse of March 202015

Noll, C.E. The Crustal Dynamics Data Information System a resource to support scientific analysis using space geodesy. $A d v$. Space Res., 45, 421-1440, 2010.

Pal, S., S.K. Chakrabarti, and S.K. Mondal. Modeling of subionospheric VLF signal perturbations associated with total solar eclipse, 2009 in Indian subcontinent. Adv. Space Res., 50, 196-204, 2012.

Pitout, F., P.L. Blelly, and D. Alcaydé. High-latitude ionospheric response to the solar eclipse of 1 August 2008: EISCAT observations and TRANSCAR simulation. J. Atmos. Sol. Terr. Phys., 105-106, 336-349, 2013.

Rashid, Z.A.A., M.A. Momani, S. Sulaiman, M.A.M. Ali, B. Yatim, G. Fraser, and N. Sato. GPS ionospheric TEC measurement during the 23rd November 2003 total solar eclipse at Scott Base Antarctica. J. Atmos. Sol. Terr. Phys., 68, 1219-1236, 2006.
Reinisch, B.W., and I.A. Galkin. Global Ionospheric Radio Observatory (GIRO). Earth, Planets and Space, 63 (4), 377-381, 2011, DOI: $10.5047 /$ eps.2011.03.001.

Tsai, H.F., and J.Y. Liu. Ionospheric total electron content response to solar eclipses. J. Geophys. Res., 104 (A6), 12657-12668, 1999.

Verhulst, T.G.W., D. Sapundjiev, and S. Stankov. High-resolution ionospheric observations and modeling over Belgium during the solar eclipse of 20 March 2015 including first results of ionospheric tilt and plasma drift measurements. Adv. Space Res., 57, 2407-2419, 2016, DOI: 10.1016/j.asr.2016.03.009.

Wenzel, D., N. Jakowski, J. Berdermann, C. Mayer, C. Valladares, and B. Heber. Global Ionospheric Flare Detection System (GIFDS). J. Atmos. Sol. Terr. Phys., 138-139, 233-242, 2016.

Cite this article as: Hoque MM, Wenzel D, Jakowski N, Gerzen T, Berdermann J, et al. Ionospheric response over Europe during the solar eclipse of March 20, 2015. J. Space Weather Space Clim., 6, A36, 2016, DOI: 10.1051/swsc/2016032. 